\title{
The Impact of Traffic Environmental Vision Pressure on Driver Behaviour
}

\author{
Yi Li $\mathbb{D}$, Yuren Chen, and Fan Wang \\ The Key Laboratory of Road and Traffic Engineering, Ministry of Education, College of Transportation Engineering, Tongji University, \\ No. 4800 Cao'an Hwy., Shanghai 201804, China \\ Correspondence should be addressed to Yi Li; smilesusanly@163.com
}

Received 27 December 2017; Revised 26 April 2018; Accepted 9 May 2018; Published 5 June 2018

Academic Editor: Giulio E. Cantarella

Copyright (C) $2018 \mathrm{Yi} \mathrm{Li} \mathrm{et} \mathrm{al.} \mathrm{This} \mathrm{is} \mathrm{an} \mathrm{open} \mathrm{access} \mathrm{article} \mathrm{distributed} \mathrm{under} \mathrm{the} \mathrm{Creative} \mathrm{Commons} \mathrm{Attribution} \mathrm{License,} \mathrm{which}$ permits unrestricted use, distribution, and reproduction in any medium, provided the original work is properly cited.

\begin{abstract}
Car-following (CF) and lane-changing (LC) behaviours are basic components in driving process. Previous models described them as physical processes with vehicle dynamics and physical criteria. However, drivers' decisions are greatly influenced by their subjective vision information of various traffic environment elements. To solve this problem, we propose a new concept of traffic environmental vision pressure to explain these two behaviours. The pressure source consists of two parts: nearby vehicles and infrastructures. Pressure models were built to quantify the impact of traffic and roadside infrastructures on these two behaviours. 103 field tests (53 LC and 50 CF) carried out by 40 drivers were conducted to test and calibrate the models. Drivers' psychological data and vehicle data were collected and postprocessed. Results showed positive relationship between drivers' psychological stress and vision pressure, which verified the assumption that traffic environmental vision information would have certain effect on driver behaviour. Quantitative thresholds of pressure value were also given and explained with test data. It is concluded that the traffic environmental vision pressure in CF and LC behaviours is quite different, and higher pressure has more impact on behaviour change. We believe that these results will be helpful to study the micro driver behaviour.
\end{abstract}

\section{Introduction}

Car-following (CF) and lane-changing (LC) are common behaviours in driving process. LC behaviour often happens when the driver cannot stand with current vehicle state. This "unsatisfactory" state will force the driver to change lane in order to improve his/her driving experience. On the other hand, if the driver is satisfied with current vehicle state, he/she will keep following, which can be observed as a CF behaviour. Based on such understanding, a lot of models have been built to explain the CF behaviour and LC behaviour.

Car-following behaviour research can be traced back to 1958 when GMR (General Motors research) model was proposed [1]. Although this model took relative speed and distance between two adjacent vehicles into consideration, it had too many assumptions for application. To overcome this weakness, TTC (time to collision) was introduced into the basic GMR model. Then it was widely used in micro driving state prediction models [2]. However, CF behaviour is not a mechanical process. It is a comprehensive outcome of drivers' reaction time and desired following distance, so CA (collision-avoidance) model, Gipps model, and IDM (intelligent driver model) were presented [3]. To have a better description of human perceptual impact on CF, driver error [4], driver distraction [5], and driver heterogeneity [6] were included in various models. This shows that the research on interaction between driver and traffic environment has become an important part in CF behaviour analysis. In urban area or traffic congestion situation, this interaction effect is more significant, which mainly comes from the next-nearest neighbouring vehicles and visual detection $[7,8]$.

Compared with car-following behaviour, there are more dynamic factors and interaction in lane-changing behaviour. Hence, plenty of studies tried to explain this process, which can be divided into three types.

(1) Physical-criteria-based LC behaviour explanatory models. This kind of models typically used some physical factors (e.g., gap acceptance, relative speed/acceleration, and desired speed/acceleration [9]) to quantify the necessity, feasibility, and safety of LC behaviour. By dividing LC process 
into three steps (1) target lane check, (2) gap acceptance, (3) lane change [10]), gap acceptance model is widely used in traffic flow and LC studies [11-14]. Results of such models revealed that gap length, relative speed/distance, and desired speed/acceleration had significant impact on LC behaviour $[15,16]$.

(2) Probability-based LC behaviour explanatory models. This kind of models focused on solving LC probability and lane choosing problem. Utility function [17, 18], cellular automata [19-21], and lattice model [22, 23] showed good performance in potential LC behaviour prediction. Moreover, the LC behaviour probability and discrete factors were combined in modelling urban lane-changing manoeuvres. It showed better estimation results under different traffic congestion than traditional models [24].

(3) Driver-behaviour-based LC behaviour explanatory models. With the rapid development of deep learning technology, more human-driver-based subjective factors can be included in the model. For example, drivers' uncertainties and perceptions are dominating factors for $\mathrm{LC}$ behaviour, but how to quantify their impact is quite difficult [25]. In latest studies, they were considered in lane-changing trajectory prediction through multilayer neural network [26]. Results showed that information from test car's neighbourhood was crucial for LC trajectory prediction. This uncertainty state can also be quantified by Tree-Augmented Naive Bayesian classifier [27]. Similar behaviour analysis can be found in LC intention recognition model [28] and lane departure prediction model [29].

Studies above have got a large number of achievements in methodology and relationship explanation between behaviour indexes and LC phenomenon. However, these behaviour indexes were actually the vehicle's status data, and they cannot directly describe the impact of traffic environment on driver behaviour. In this paper, we believe that a human-driver-based behaviour explanatory model needs to explain the original reason for such behaviour from drivers' view [30]. Based on this concept, a vision-based LCDAS (Lane Change Decision Aid System) was established to detect vehicles and motorcycles following behind in adjacent lanes with a single camera. Hence, it could warn drivers of dangerous situations [31]. Traffic environment details, such as lane line [32], vehicle type [33], vehicle on current, and adjacent lane $[34,35]$ were proved to have close relationship with LC behaviour. Recently, large volumes of realworld driving video and vehicle status have been collected through naturalistic driving program in USA, Europe, and China. Corresponding studies showed that driver would have a series of object searching and checking actions before, during, and after LC behaviour [36, 37]. These results can be regarded as an indirect way to understand the impact of traffic environment on driver behaviour. Nevertheless, drivers' main source of traffic information is their vision instead of mechanically scanning data or remote video surveillance. To overcome this problem, a trade-off theory was introduced to explain the reason of LC behaviour. This theory described it as a trade-off between the expected own advantages (test vehicle) and disadvantages of others (other vehicles). A safe lane-changing behaviour must have more merits than a car-following behaviour [38]. Following this study, a road vision information model was built to classify the traffic environmental information. This provided a basic method to explain vision information in drivers' view [39]. Furthermore, driver perspective principle in road alignment vision model successfully distinguished the difference between vision perception information and physical criteria [40].

Past studies showed great improvement in CF/LC behaviour modelling and driver characteristics analysis. However, the influence mechanism of traffic environment on such behaviour still needs a systematic theory based on drivers' subjective vision information, which may become the foundation of personified autopilot system. To interpret this mechanism, we assume that driver behaviour is motivated by the vision pressure that comes from nearby vehicles and roadside infrastructures. In this paper, we mainly define, model, and test the traffic environmental vision pressure.

This paper is organized as follows. Section 1 introduces the achievements and weaknesses of past studies on CF and LC behaviour models and traffic environment vision information models. Section 2 defines the traffic environmental vision pressure and its sources. Section 3 describes the pressure model and its parameters in detail. Section 4 explains the field experiments. Section 5 shows the test results and verification results of the pressure model. Section 6 concludes the main achievements and limitations of the paper.

\section{Definition of the Traffic Environmental Vision Pressure}

As a part of "road-environment-driver" system, driver is not only the producer of various behaviours, but also the receiver of massive traffic environment information. To explain the impact of such phenomenon, we assume that during a driving task, driver will receive different real-time traffic environment information through their vision. The information will form a certain inner pressure on driver, which comes from two sources: vehicles around the test vehicle and infrastructures near the test vehicle (see Figure 1).

In this research, driver behaviour is regarded as the consequence of perception, reaction, and decision based on traffic environmental information, so the behaviour motivation can be described as traffic environmental pressure. In a safe driving process, a driver would drive properly to keep the pressure stable (e.g., car-following). If the pressure is too high to keep following, he/she will seek a behaviour change to release or lower the overall pressure. This is the ultimate reason of various behaviours. Figure 2 shows an example of CF and LC motivated by traffic environmental vision pressure.

\section{Traffic Environmental Vision Pressure Model}

Traffic environmental vision pressure is a comprehensive expression of traffic effect on driver. In a normal driving process, a driver makes a decision based on their vision 


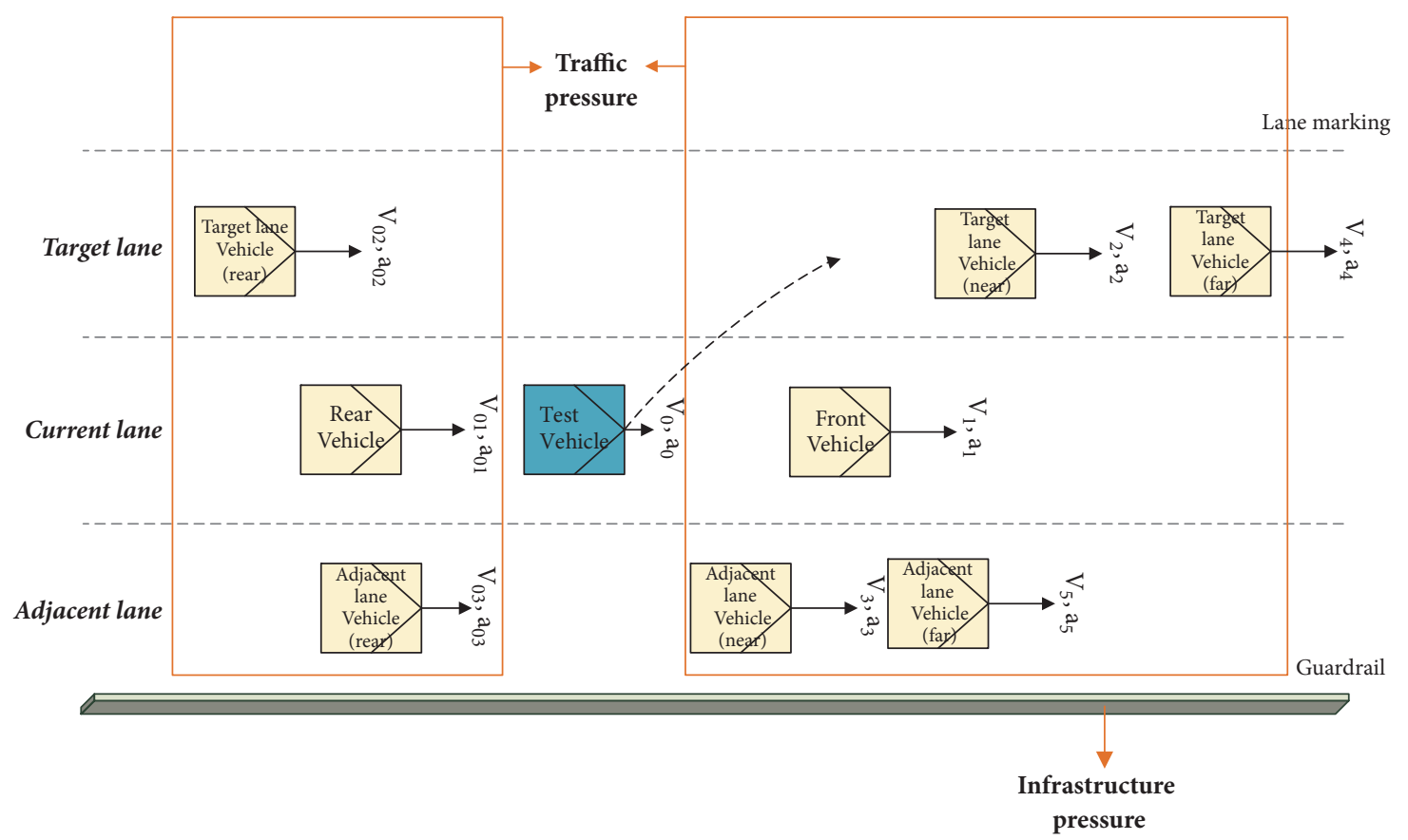

(a)

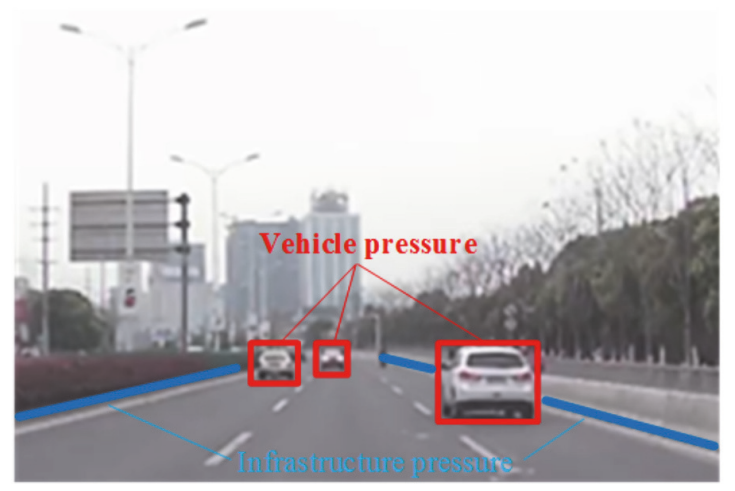

Front view

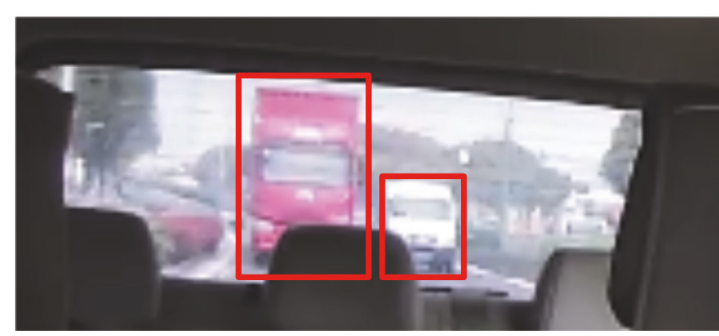

Rear view

(b)

FIgURE 1: Two sources of traffic environmental vision pressure ((a) top view, (b) first perspective view).

information, such as the speed and acceleration of nearby vehicles, as well as the constraint of roadside infrastructures. According to Chinese Design Specification for Highway Alignment (JTG D20-2006), proper safe distance and relative speed are important factors for safe driving. Therefore, the vision pressure consists of two parts: vehicle pressure and road infrastructure pressure.

\section{(1) Vehicle Vision Pressure}

(i) Speed Constraint. The speed factor $(\sigma)$ reflects the relative speed between test vehicle and nearby vehicle.

$$
\sigma=v_{0}-v_{n}
$$

where $v_{0}$ is the speed of test vehicle and $v_{n}$ is the speed of nearby vehicle $(n)$.
The acceleration factor $(\eta)$ reflects the relative acceleration between test vehicle and nearby vehicle.

$$
\eta=a_{0}-a_{n}
$$

where $a_{0}$ is the acceleration of the test vehicle and $a_{n}$ is the acceleration of nearby vehicle $n$.

(ii) Distance Constraint. The distance constraint factor $(S)$ reflects drivers' tolerance of gap according to current vehicle status.

$$
S=\sigma \cdot d t+\frac{1}{2} \cdot \eta \cdot d t^{2}
$$

where $d t$ is the time interval of data collection. 

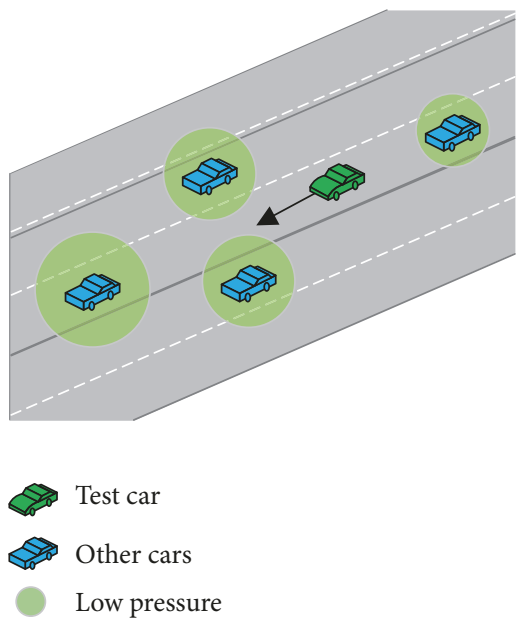

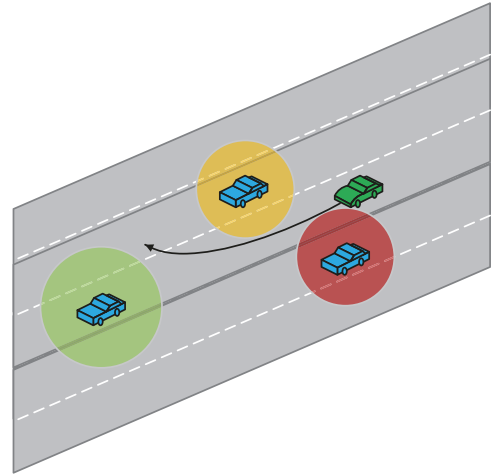

Medium pressure

Low pressure

Other cars

High pressure

(b)

FIGURE 2: Illustration of CF behaviour (a) and LC behaviour (b) motivated by traffic environmental vision pressure.

Based on the above constraints, we can calculate the vision pressure (nondimensional) of nearby vehicle as follows:

$$
F_{v}=\frac{S}{R_{n}}+1
$$

where $R_{n}$ (scalar) is the relative distance between test vehicle and nearby vehicle in drivers' vision.

In this formula, the pressure reflects the relationship of space supply between test vehicle and nearby vehicle. The demand part is $S$, which shows how much space the test vehicle needs to follow the target vehicle $(n)$ in the same speed. The supply part is $R_{n}$, which is the objective space status around test vehicle. Therefore, if the demand distance is much larger than the supply space, then the vehicle pressure would be large. Corresponding mathematical expression is as follows:

$$
\begin{aligned}
F_{v}= & \frac{S}{R_{n}} \\
+1 & \begin{cases}>1, & \text { if demand } S \text { is larger than supply } R_{n}, \\
=1, & \text { if demand } S \text { is equal to supply } R_{n}, \\
<1, & \text { if demand } S \text { is smaller than supply } R_{n} .\end{cases}
\end{aligned}
$$

This formula shows the qualitative relationship between demand and supply for test vehicle. For detail analysis, quantitative threshold needs to be calibrated with field tests (see Section 5).

(2) Road Infrastructure Vision Pressure. Similar to the nearby vehicle vision pressure formula, infrastructure vision pressure consists of three parts: (1) latitudinal speed constraint,
(2) latitudinal distance constraint, and (3) guardrail type. Corresponding pressure formula is as follows:

$$
F_{i}=\frac{v_{\text {lat }}^{2}}{2 R_{i} a_{\text {lat }}} \cdot m,
$$

where $F_{i}$ is the infrastructure vision pressure (nondimensional); $v_{\text {lat }}$ is the latitudinal speed of test vehicle; $a_{\text {lat }}$ is the latitudinal acceleration of test vehicle; $R_{i}$ is the distance between test vehicle and infrastructure; and $m$ is the type of nearby infrastructure $(1=$ lane marking, $1.5=$ concrete pier/metal fence, 2 = green belt).

Figure 3 illustrates the impact of different pressure sources on test vehicle and their integrated effect. Each element in drivers' view has a certain pressure on driver. As shown in Figure 3, resultant vision pressure consists of the pressure from current lane, target lane, adjacent lane, and guardrail. Each pressure forces the driver to "keep away", so high pressure is more dominating than low pressure on drivers' decision. For instance, the direction of resultant vision pressure $(\Sigma F)$ on test vehicle in Figure 3 is "left-back". This pressure forces driver to slow down and make a left lane change to release the pressure, which is consistent with expectation.

\section{Experiments and Data}

In order to quantify the impact of vision pressure on driver behaviour, we conducted a series of field experiments to obtain real-time vehicle data and driver behaviour data. Several types of equipment and sensors were used in the field tests. Detailed experimental methods are described as follows.

4.1. Participants and Scenes. A total of 40 drivers who had over 3-year driving experience took part in the experiments. 
TABLE 1: Test route illustration.

\begin{tabular}{lcccc}
\hline Road type & Lane (lane/direction) & Lane width $(\mathrm{m})$ & Speed limit $(\mathrm{km} / \mathrm{h})$ & Median strip \\
\hline Arterial road & 3 & 3.75 & 80 & Green belt \\
Urban street & 2 & 3.75 & 40 & Guardrail \\
\hline
\end{tabular}

Lane marking

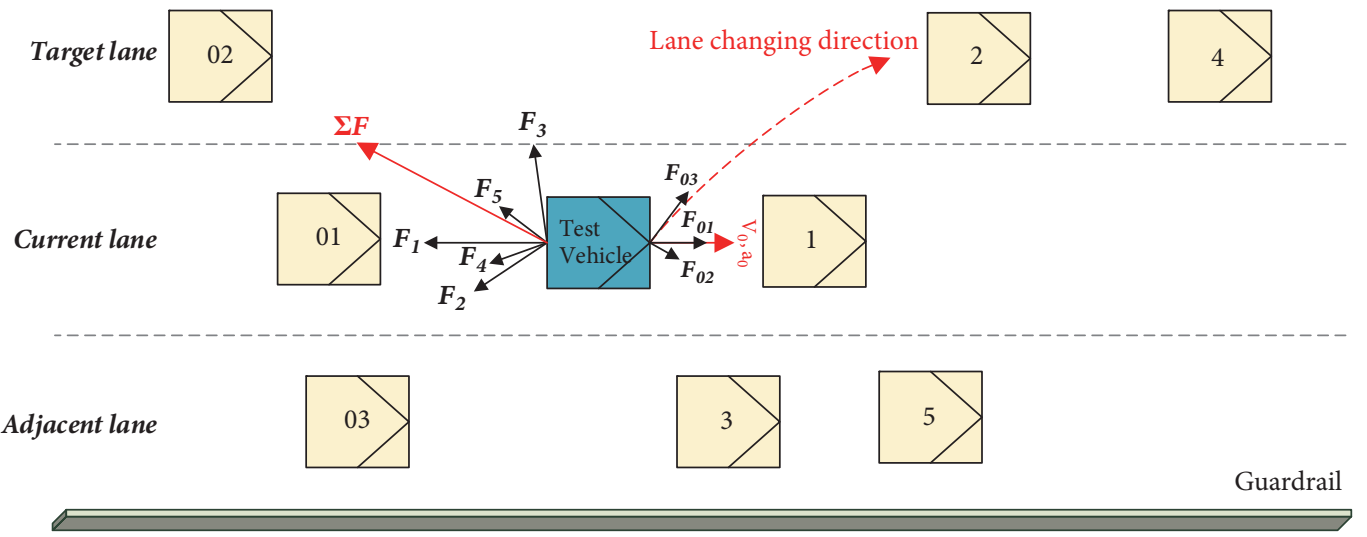

FIGURE 3: Impact of resultant vision pressure $(\Sigma F)$ on vehicle.

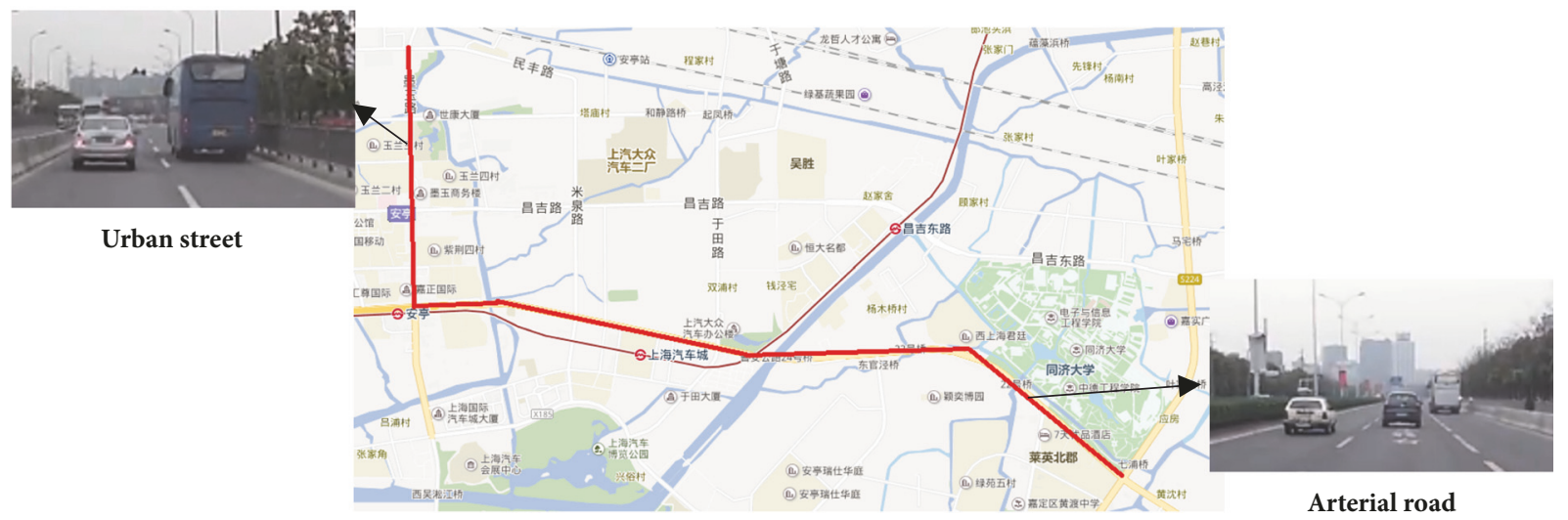

FIGURE 4: Test route and scenes.

They were between the age of 30 and 40 (mean $=36.2$, SD $=3.5$ ). Drivers were asked to drive a sedan along the test route without tasks (no time limit, no observer) in sunny days. Each driver drove the test vehicle for a while to get familiar with its control system before tests. During the tests, participants could follow or change lane according to their own decisions. Speeding was not allowed in the tests. Equipment was preinstalled on the test vehicle. The test route consisted of an arterial road and an urban street, located in Jiading District, Shanghai, China. Along the test route, there were ten traffic lights, one university and one factory. The route and scenes are shown in Figure 4 and Table 1.

4.2. Equipment and Data Collection. To collect real vision data and driver behaviour during LC, we installed on-board recording devices on test vehicle. Each device was checked so as not to block drivers' vision and their body movement.
Three types of data recording devices (see Figure 5) were applied in the tests, including a set of physiological monitoring equipment, a driving recorder, and a binocular camera.

(1) Physiological monitoring equipment (VARIOPORT) collects drivers' heart rate signal during the driving process.

(2) Driving recorder (GARMIN GDR 35) collects traffic environment image (front and rear) and real-time vehicle data, including speed, triaxial acceleration, longitude, and latitude.

(3) Binocular camera collects depth of field data of image. Corresponding data explanations are listed in Table 2.

The relative distance is collected automatically by binocular camera according to binocular stereo vision algorithm (supported by Intel RealSense Technology). HF is the sum of high frequency $(0.15-0.40 \mathrm{~Hz})$ of HRV (Heart Rate Variability), which is related to sympathetic nerve activity and tension. LF is the sum of low frequency $(0.04-0.15 \mathrm{~Hz})$ of 


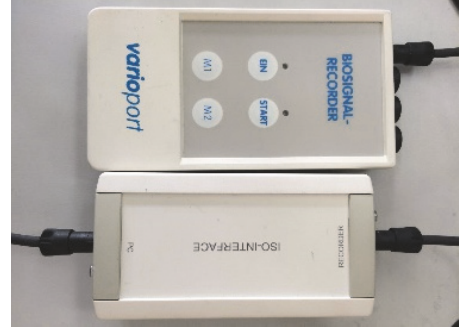

(a)

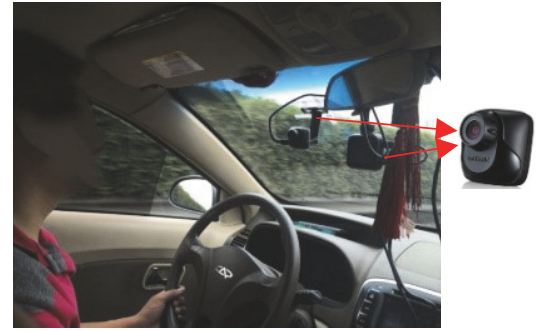

(b)

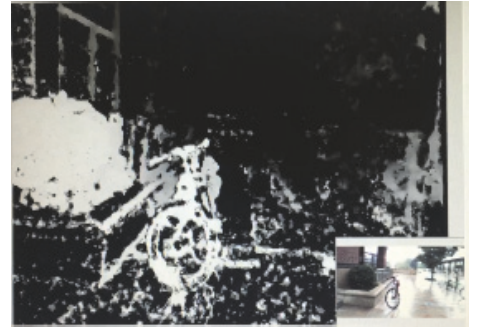

(c)

FIgURE 5: Experiment equipment. (a) Physiological monitoring equipment. (b) Driving recorder (GARMIN GDR 35). (c) Depth of field data.

TABLE 2: Data collection illustration.

\begin{tabular}{lccc}
\hline Data source & Name & Accuracy & Frequency \\
\hline & Vertical acceleration & $0.001 \mathrm{~g}$ & $60 \mathrm{~Hz}$ \\
Driving recorder & Longitudinal acceleration & $0.001 \mathrm{~g}$ & $60 \mathrm{~Hz}$ \\
& Latitudinal acceleration & $0.001 \mathrm{~g}$ & $\mathrm{~Hz}$ \\
& Speed & $1 \mathrm{~km} / \mathrm{h}$ & $1 \mathrm{~m}$ \\
\hline \multirow{2}{*}{ Binocular camera } & Relative distance & $1 \mathrm{~km} / \mathrm{h}$ & $/$ \\
\hline & Relative speed & $0.001 \mathrm{mv}$ & $1 \mathrm{~ms}^{2}$ \\
Physiological monitoring equipment & ECG (Electrocardiograph) & $1 \mathrm{~ms}^{2}$ & $/$ \\
\hline
\end{tabular}

Note. "g" means gravity $\left(9.8 \mathrm{~m} / \mathrm{s}^{2}\right)$.

HRV, which is related to parasympathetic nerve activity and relaxation. The ratio of LF and HF reflects the balance of sympathetic nerve and parasympathetic nerve activity [41, 42].

\section{Results and Analysis}

In this paper, we focus on three types of behaviour: carfollowing (Type 0), left lane-changing (Type 1), and right lane-changing (Type 2). Each type was classified manually according to on-board video. The raw data were cleansed according to two principles: (1) discarding mandatory lane change behaviour (e.g., entering and exiting the intersection); (2) deleting discontinuous behaviour records. Then we have 103 tests (53 lane-changing and 50 car-following) valid for this research.

As mentioned in Section 3, the vision pressure varies with the change of nearby traffic environment, so the resultant pressure also changes during a $\mathrm{CF} / \mathrm{LC}$ behaviour. As LC behaviour is more complex than CF behaviour, we divided it into three phases: before LC (B-LC), during LC (D-LC), and after LC (A-LC).

(i) B-LC: before changing lane, the driver has collected a lot of traffic environmental information for a while, which forms a series of vision pressure. If the vision pressure is too high for the driver to keep the current vehicle status, then he/she will change lane to get a more harmonious pressure environment. (ii) D-LC: when changing from the current lane to the target lane, the driver will face a dynamic pressure environment. It is a pressure release process, but it may also be accompanied by some pressure increase.

(iii) A-LC: after changing to the target lane, the pressure change still goes on with the change of nearby vehicles and infrastructure, which means the high vision pressure (if existing) will not decrease immediately. Therefore, a steady A-LC phase ensures a safe LC process.

Correspondingly, the vision pressure of each vehicle and infrastructure around the test vehicle were calculated according to the model proposed in Section 3. Table 3 listed the corresponding explanation.

First of all, as the base of vision pressure theory, the relationship between traffic environmental vision pressure and drivers' inner psychological stress needs to be proved. The statistical results of drivers' psychology index (LF/HF) and pressure are shown in Table 4.

It shows that the ratio of LF and HF during carfollowing behaviour is lower than that during lane-changing behaviour, but the psychological stress of different lanechanging direction is quite the same. This means that high vision pressure would result in high psychological stress. Figure 6 visualizes the relationship between resultant vision pressure and psychology index.

Figure 6 shows that the resultant vision pressure is positively correlated to psychology index (correlation index $=0.71$ ). It also verifies that the traffic environmental information or scene has a certain impact on drivers' psychology 


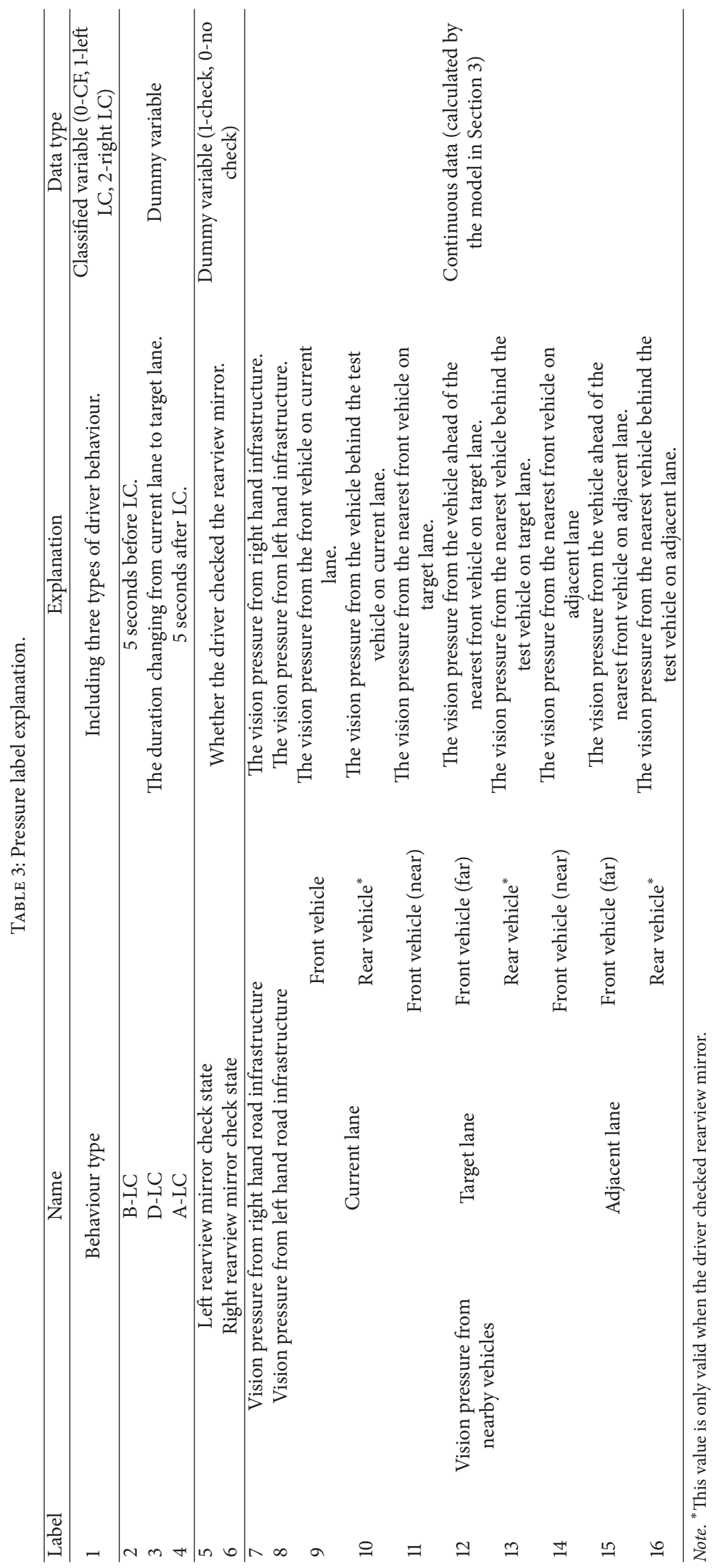


TABLE 4: Statistical results of average pressure and LF/HF.

\begin{tabular}{|c|c|c|c|c|c|c|}
\hline \multirow{2}{*}{ Behaviour type } & \multirow{2}{*}{ LF/HF } & \multicolumn{2}{|c|}{ Pressure from road infrastructure } & \multicolumn{3}{|c|}{ Pressure from nearby vehicles } \\
\hline & & Right-hand & Left-hand & Current lane & Target lane & Adjacent lane \\
\hline Car following & 1.38 & 5.66 & 8.42 & 0.32 & 0.64 & 0.25 \\
\hline \multicolumn{7}{|l|}{ Left LC } \\
\hline B-LC & & 9.43 & 8.38 & 5.63 & 1.93 & 1.72 \\
\hline D-LC & 1.46 & 7.74 & 6.67 & 3.88 & 1.28 & 1.42 \\
\hline A-LC & & 3.32 & 6.42 & 1.33 & 1.33 & 1.11 \\
\hline \multicolumn{7}{|l|}{ Right LC } \\
\hline B-LC & & 9.98 & 14.56 & 4.11 & 1.77 & 1.25 \\
\hline D-LC & 1.47 & 8.19 & 9.32 & 3.62 & 1.43 & 1.07 \\
\hline A-LC & & 11.98 & 7.19 & 1.57 & 2.27 & 1.07 \\
\hline
\end{tabular}

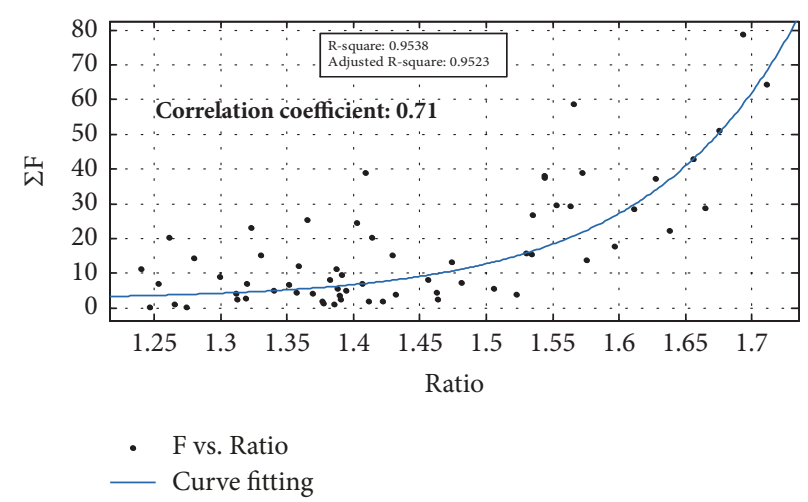

Figure 6: Relationship between vision pressure $(\Sigma F)$ and psychology index (LF/HF).

and behaviour, which is interpreted as vision pressure in this paper.

Secondly, the field tests provided quantitative data for pressure threshold calibration. According to all the pressure results calculated by (5) and (6), we divided the pressure set into four stages with three thresholds.

Stage $1(F \geq 5)$. This threshold is the average pressure of front vehicle on current lane before lane-changing behaviour. Pressure larger than 5 means that the resistance force is too large for the test vehicle to keep following.

Stage $2(1<F<5)$. Pressure in this interval means that the stress does exist but is affordable for test vehicle. If the pressure keeps climbing up, there will be a potential behaviour change.

Stage $3(0.4<F \leq 1)$. The threshold 0.4 is the average value of vehicle pressure during car-following behaviour, and threshold 1 is the critical value that represents the balance state between space demand and supply. Pressure in this interval means that the test vehicle gets no intense stress from nearby vehicle, and there is some free space for flexible driving.

Stage $4(F<0.4)$. If the pressure is lower than 0.4 , then the nearby vehicles and infrastructure may have attractive effect on test vehicle. In such state, the test vehicle has much free space to keep following and the probability of lane-changing behaviour is small.

With these quantitative stages and positive correlation between vision pressure and driver psychological stress, we can further analyze the impact of vision pressure distribution in CF and LC behaviour. The pressure results are shown in Figures 7 and 8. During a car-following behaviour, target lane means left-hand lane and adjacent lane means right-hand lane. It can be seen from Figure 7 that pressure from road infrastructure (especially the left side) has dominant impact on car-following behaviour. Among the nearby vehicles, the test vehicle appears to be more influenced by the front vehicle on left-hand lane. The pressure from the front vehicle on current lane is quite low, and in some tests it is even below 0.4 . Besides, we can see that some pressure values in Figures 7 and 8 are even below zero. This results from the lower speed or acceleration of test vehicle compared with nearby vehicles. In such situation, according to (3), the value of $S$ is negative. If the absolute value of $S$ is much larger than $R_{n}$, then the pressure value would also be negative, which means that the nearby vehicles have large attraction impact on test vehicle. In this situation, driver is satisfied with current vehicle status and the front vehicle even has an attractive effect on test vehicle. This explains the reason why the driver keeps following in such pressure circumstance.

Figure 8 shows the vision pressure comparison between left LC (Type 1) and right LC (Type 2) in three phases. 


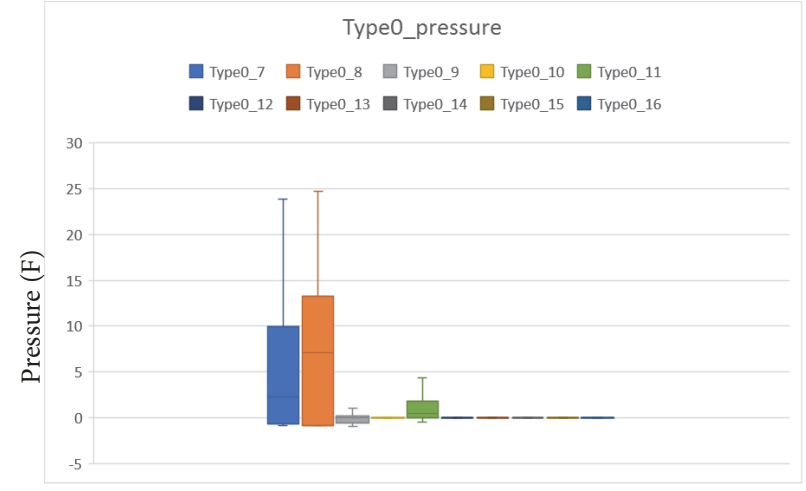

FIgURE 7: Vision pressure of car-following (the numbers in the legend refer to Table 3).

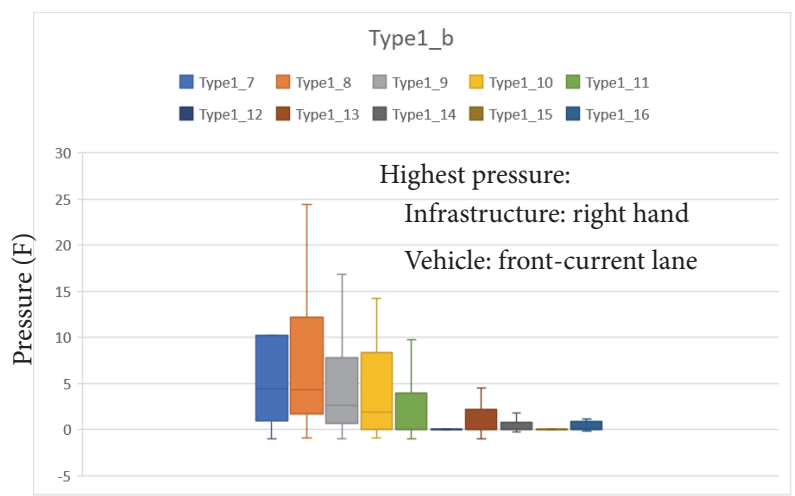

(a) Pressure before left lane change

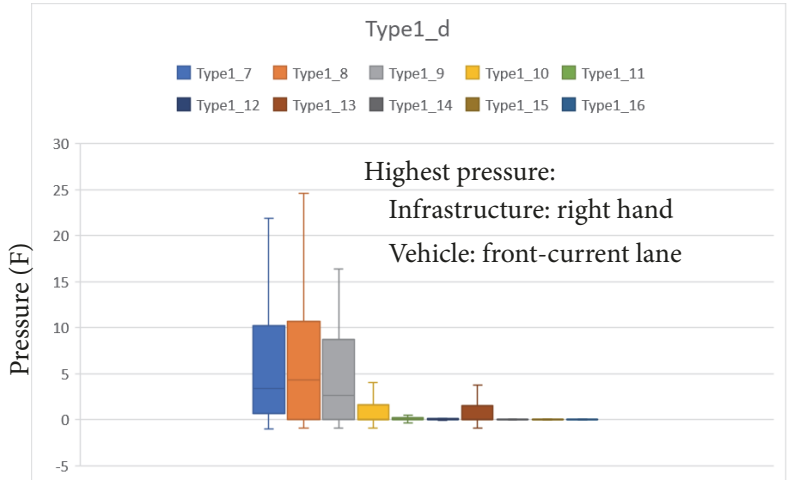

(c) Pressure during left lane change

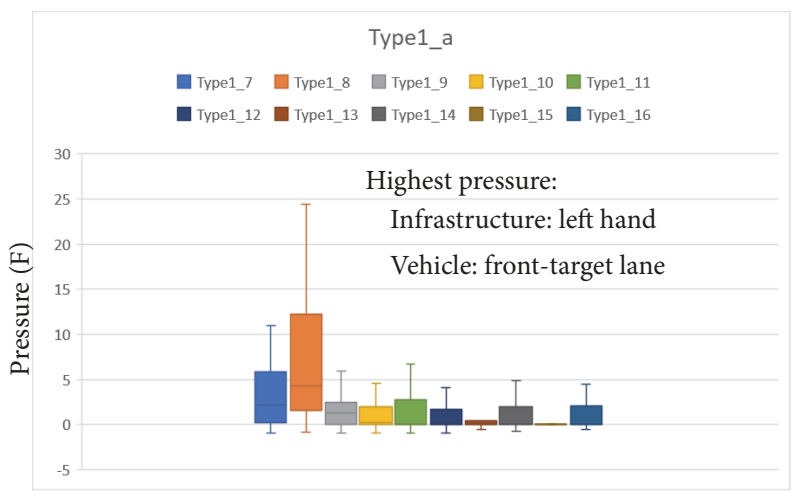

(e) Pressure after left lane change

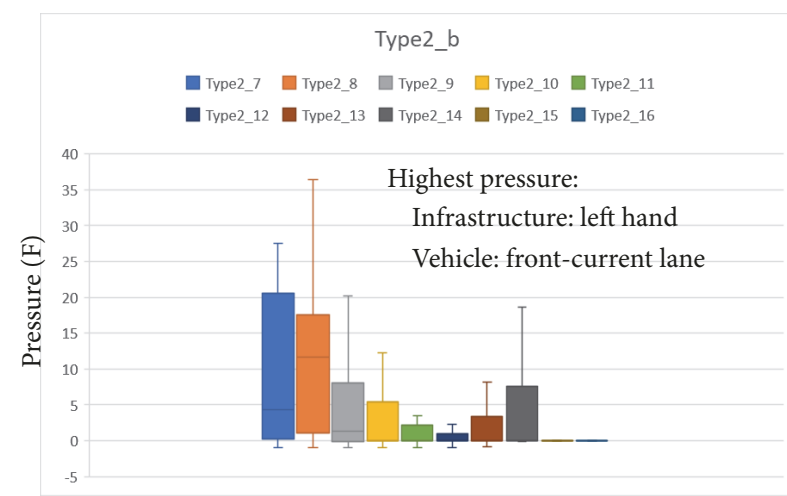

(b) Pressure before right lane change

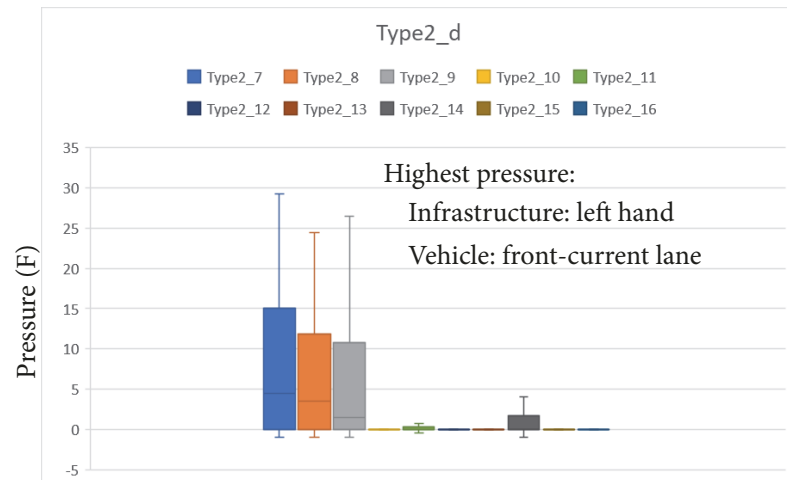

(d) Pressure during right lane change

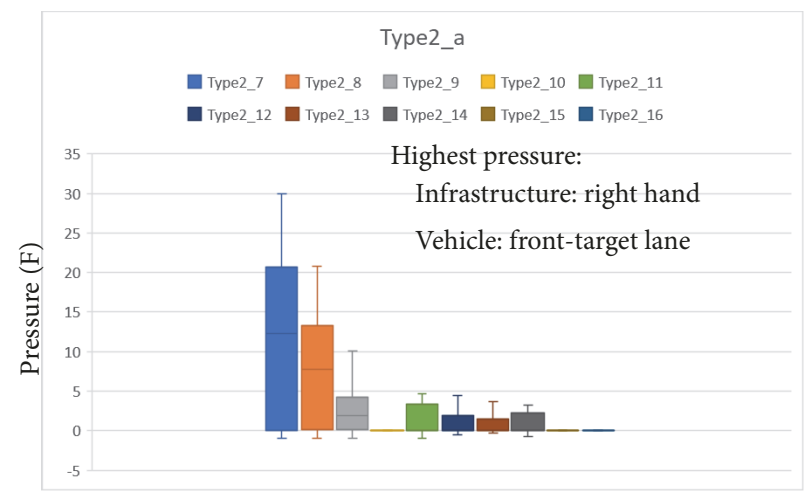

(f) Pressure after right lane change

Figure 8: Vision pressure of lane-changing behaviour (the numbers in the legend refer to Table 3). 
In B-LC phase, road infrastructure vision pressure has the most significant impact for both behaviour types, and the infrastructure pressure from the opposite direction is more dominating. This implies that driver will check the infrastructure pressure on opposite side before changing lane. As for the vehicle vision pressure, the vehicles ahead and behind on current lane are obviously the main factors for drivers' LC decision. Insufficient space supply of vehicle ahead has negative effect on test vehicle. Meanwhile, the following vehicle has "push" effect on test vehicle. In left B-LC, the near front and rear vehicles on target lane also have great impact on test vehicle. This phenomenon is in accordance with gap acceptance theory: drivers pay attention to traffic status of target lane before changing lane to ensure a safe lane-changing behaviour. Although the pressure from adjacent lane is relative low, the total pressure (including the road infrastructure pressure) is mainly influenced by the right-hand resultant force. In right B-LC, the pressure from near front vehicle on adjacent lane is higher than the pressure from target lane. Therefore, the existence of this high pressure will possibly force drivers to change to the opposite lane.

In D-LC phase, the vision pressure is generally lower than the pressure in B-LC phase. The pressure from road infrastructure is still the most important element. When changing from current lane to target lane, the pressure from front vehicle on current lane shows an increment with decrease of relative distance and increase of relative speed. The pressure from near front vehicle on adjacent lane is the next high pressure element in this phase. The average values are 2.64 and 2.54 for left LC and right LC, respectively. The third highest pressure in Figure 8(c) shows the resistant impact of the coming rear vehicle on target lane on test vehicle.

After finishing the LC behaviour, the pressure distribution will not drop immediately which is proved in Figures $8(\mathrm{~d})$ and $8(\mathrm{f})$. The nearby vehicle vison pressure turns to be relatively equal in A-LC phase, but the pressure from road infrastructure is still high and unbalanced. This state has a positive relationship with the direction of lane-changing behaviour. In other words, a left LC behaviour is followed by a dominating pressure from the same direction of road infrastructure.

\section{Conclusions}

This paper puts forward a novel concept of traffic environmental vision pressure to explain the reason of lanechanging behaviour. The vision pressure comes from two aspects: vehicles and infrastructures, which can be quantified by the presented vision pressure model. In the vehicle vision pressure model, speed constraint and distance constraint are important factors. In the road infrastructure vision pressure, latitudinal speed constraint, latitudinal distance constraint, and guardrail type are considered.

A series of field tests were conducted to collect drivers' real-time psychological and driving data. The correlation result shows positive relationship between drivers' psychological stress and vision pressure, which confirms the hypothesis that traffic environment has certain effect on drivers' inner stress. However, drivers' psychological stress is not easy to collect in normal driving process, so the impact of vision pressure in CF behaviour and LC behaviour (divided into three phases: before, during, and after) is deeply discussed. It shows that the resultant vision pressure will determine an LC behaviour and its direction. For example, compared with vision pressure of car-following behaviour, drivers' pressure during lane-changing behaviour is much higher. This explains the critical issue discussed by Zheng [25] that how to measure the impact of traffic characteristics on human decision-making process is important to precisely predict driver behaviour. Among all types of vision pressure, the road infrastructure determines vehicles' moving direction and driving rules. Therefore, proper design and assignment of roadside infrastructure will help driver to drive more safely. On the other hand, the result shows that improper vision pressure from the front and rear vehicles on current lane is the most dominating factor for lane-changing. The vehicles on target lane and adjacent lane also influence drivers' LC decision before action, but the impact from the former continuously works throughout the whole LC process, which confirms and extends the conclusions in Moridpour et al's and Oh et al's studies [34, 43].

Apart from the achievements above, this study still has some limitations in the following aspects. Firstly, driving process is a continuous state, so drivers receive changing pressure continuously. This kind of pressure change is a time related process, which means that transient pressure is a state criterion. For the data limitation, this paper only focuses on CF and LC behaviour separately and does not consider these two behaviours as a circulation. Secondly, drivers' psychological stress may also be influenced by many other factors, such as motion, genders, and healthiness. The psychological factor used in this paper is an integrated concept and all participants are healthy and experienced drivers, so there will be limits in the generalization of the vision pressure model. Thirdly, the test vehicle is a sedan, so the video records and vehicle data are only valid for similar vehicles. The pressure distribution needs to be reconsidered for other vehicle types (e.g., truck or semitrailer), because different vehicle types may result in different drivers' vision angles.

In the following studies, we will not only overcome the limits mentioned above, but also try to quantify the pressure changing process and its accumulative impact on lane-changing and overtaking behaviour. We believe that these studies may present a further understanding of the impact of traffic environment on driver behaviour.

\section{Conflicts of Interest}

The authors declare that they have no conflicts of interest regarding the publication of this paper.

\section{Acknowledgments}

This work was supported by the National Science Foundation of China [Grant no. 51238008]. 


\section{References}

[1] R. E. Chandler, R. Herman, and E. W. Montroll, "Traffic dynamics: studies in car following," Operations Research, vol. 6, pp. 165-184, 1958.

[2] S. Jin, Z.-Y. Huang, P.-F. Tao, and D.-H. Wang, "Car-following theory of steady-state traffic flow using time-to-collision," Journal of Zhejiang University SCIENCE A, vol. 12, no. 8, pp. 645-654, 2011.

[3] M. Saifuzzaman and Z. Zheng, "Incorporating human-factors in car-following models: a review of recent developments and research needs," Transportation Research Part C: Emerging Technologies, vol. 48, pp. 379-403, 2014.

[4] N. A. Stanton and P. M. Salmon, "Human error taxonomies applied to driving: A generic driver error taxonomy and its implications for intelligent transport systems," Safety Science, vol. 47, no. 2, pp. 227-237, 2009.

[5] M. A. Regan, J. D. Lee, and K. Young, Driver Distraction: Theory, Effects and Mitigation "Automobile Drivers", 2008.

[6] H. Farah, H. N. Koutsopoulos, M. Saifuzzaman, R. Kölbl, S. Fuchs, and D. Bankosegger, "Evaluation of the effect of cooperative infrastructure-to-vehicle systems on driver behavior," Transportation Research Part C: Emerging Technologies, vol. 21, no. 1, pp. 42-56, 2012.

[7] M. Treiber, A. Kesting, and D. Helbing, "Delays, inaccuracies and anticipation in microscopic traffic models," Physica A: Statistical Mechanics and its Applications, vol. 360, no. 1, pp. 7188, 2006.

[8] E. Muhrer and M. Vollrath, "The effect of visual and cognitive distraction on driver's anticipation in a simulated car following scenario," Transportation Research Part F: Traffic Psychology and Behaviour, vol. 14, no. 6, pp. 555-566, 2011.

[9] P. G. Gipps, "A model for the structure of lane-changing decisions," Transportation Research Part B: Methodological, vol. 20, no. 5, pp. 403-414, 1986.

[10] Q. Yang, "A simulation laboratory for evaluation of dynamic traffic management system," Simulation, 1997.

[11] L. G. H. Fortuijn and S. P. Hoogendoorn, "Capacity estimation on turboroundabouts with gap acceptance and flow level methods," Transportation Research Record, vol. 2517, pp. 71-79, 2015.

[12] H. A. Ghods and F. F. Saccomanno, "Microscopic overtaking gap acceptance model for two-lane highways," in Proceedings of the Transportation Research Board 93rd Annual Meeting, 2014.

[13] V. Milanes, J. Godoy, J. Villagra, and J. Perez, "Automated onramp merging system for congested traffic situations," IEEE Transactions on Intelligent Transportation Systems, vol. 12, no. 2, pp. 500-508, 2011.

[14] A. Kondyli and L. Elefteriadou, "Modeling driver behavior at freeway-ramp merges," Transportation Research Record Journal of the Transportation Research Board, vol. 2249, pp. 29-37, 2011.

[15] K. I. Ahmed, M. Ben-Akiva, H. N. Koutsopoulos, and R. G. Mishalani, "Models of freeway lane changing and gap acceptance behaviour," in Proceedings of The 13th International Symposium on Transportation and Traffic Theory, Lyon, France, July 1996.

[16] M. Brackstone, M. Mcdonald, and J. Wu, "Lane changing on the motorway: factors affecting its occurrence, and their implications. Road transport information and contro," in Proceedings of the International Conference on IET, pp. 160-164, 1998.

[17] K. I. Ahmed, Modeling Drivers' Acceleration and Lane Changing Behaviour, Massachusetts Institute of Technology, 1999.
[18] T. Toledo, C. F. Choudhury, and M. E. Ben-Akiva, "Lanechanging model with explicit target lane choice," Transportation Research Record: Journal of the Transportation Research Board, vol. 1, no. 1934, pp. 157-165, 2005.

[19] E. Fukuda, J. Tanimoto, Y. Iwamura et al., "Field measurement analysis to validate lane-changing behaviour in a cellular automaton model," Physical Review E, vol. 94, no. 5, 2016.

[20] Q. Wang, "Simulation on influencing of lane changing probability on traffic flow base on cellular automata," Journal of Information \& Computational Science, vol. 10, no. 9, pp. 25612567, 2013.

[21] X. G. Li, B. Jia, Y. Z. Gao et al., "A realistic two-lane cellular automata traffic model considering aggressive lane-changing behaviour of fast vehicle," Physica A Statistical Mechanics \& Its Applications, vol. 367, no. (C), pp. 479-486, 2006.

[22] G. H. Peng, X. H. Cai, C. Q. Liu, and M. X. Tuo, "A new lattice model of traffic flow with the anticipation effect of potential lane changing," Physics Letters A, vol. 376, no. 4, pp. 447-451, 2012.

[23] Z. Li, R. Zhang, S. Xu, and Y. Qian, "Study on the effects of driver's lane-changing aggressiveness on traffic stability from an extended two-lane lattice model," Communications in Nonlinear Science and Numerical Simulation, vol. 24, no. 1-3, pp. 52-63, 2015.

[24] D. Sun and L. Elefteriadou, "Research and implementation of lane-changing model based on driver behavior," Transportation Research Record, no. 2161, pp. 1-10, 2010.

[25] Z. Zheng, "Recent developments and research needs in modeling lane changing," Transportation Research Part B: Methodological, vol. 60, pp. 16-32, 2014.

[26] R. S. Tomar, S. Verma, and G. S. Tomar, "Prediction of lane change trajectories through neural network," in Proceedings of the International Conference on Computational Intelligence and Communication Networks, pp. 249-253, 2011.

[27] F. Yan, M. Eilers, A. Ludtke, and M. Baumann, "Developing a model of driver's uncertainty in lane change situations for trustworthy lane change decision aid systems," in Proceedings of the 2016 IEEE Intelligent Vehicles Symposium, (IV 2016), pp. 406-411, IEEE, June 2016.

[28] C. Wu, Y. Li, X. Ma, H. Zhang, and H. Li, "A recognition model for lane change intention based on neural network," in Proceedings of the 2nd International Conference on Transportation Information and Safety: Improving Multimodal Transportation Systems - Information, Safety, and Integration, ICTIS 2013, pp. 125-130, July 2013.

[29] X. Wang, L. M. Yi, and D. S. Kochhar, "MTS-DeepNet for lane change prediction," in Proceedings of the International Joint Conference on Neural Networks, pp. 4571-4578, 2016.

[30] R. Poppe, "A survey on vision-based human action recognition," Image and Vision Computing, vol. 28, no. 6, pp. 976-990, 2010.

[31] Y. E. Chung, C. H. Jung, E. Chang et al., "Vision Based for Lane Change Decision Aid System," in Proceedings of the International Forum on Strategic Technology, pp. 10-13, IEEE, 2006.

[32] C. Guo, K. Kidono, and Y. Kojima, "Understanding surrounding vehicles in urban traffic scenarios based on a low-cost lane graph," in Proceedings of the IEEE Intelligent Vehicles Symposium, (IV'15), pp. 511-518, IEEE, July 2015.

[33] F. Ran, Z. Jiang, and M. Xu, "Vision-based lane detection algorithm in urban traffic scenes," Communications in Computer and Information Science, vol. 463, pp. 409-419, 2014. 
[34] S. Moridpour, G. Rose, M. Sarvi, and E. Mazloumi, "Influence of the surrounding traffic characteristics on lane changing decision of heavy vehicle drivers," Road \& Transport Research A Journal of Australian \& New Zealand Research \& Practice, vol. 21, no. 3, pp. 19-33, 2012.

[35] F. Li, L. Gao, and X. Li, "Research on lane change timing model considering the influence of four surrounding vehicles," in Proceedings of the 2014 IEEE Transportation Electrification Conference and Expo, (ITEC Asia-Pacific '14), IEEE, September 2014.

[36] L. Tijerina, W. R. Garrott, D. Stoltzfus et al., "Eye glance behavior of van and passenger car drivers during lane change decision phase," Transportation Research Record, vol. 1937, no. 1, pp. 37-43, 2005.

[37] R. K. Satzoda and M. M. Trivedi, "Drive analysis using vehicle dynamics and vision-based lane semantics," IEEE Transactions on Intelligent Transportation Systems, vol. 16, no. 1, pp. 9-18, 2015.

[38] A. Kesting, M. Treiber, and D. Helbing, "General lanechanging model for car-following model," Transportation Research Record: Journal of the Transportation Research Board, vol. 1999, no. 1, pp. 86-94, 2007.

[39] Y. J. Dong and Y. R. Chen, "An evaluation of urban traffic environment based on the amount of visual information," Journal of Transportation Information and Safety, vol. 6, no. 32, pp. 146-152, 2014.

[40] Y. R. Chen, B. Yu, and S. H. He, "Coordination between highway horizontal and vertical alignments based on drivers visual perception deviation," Journal of Tongji University (Natural Science), vol. 9, no. 32, pp. 1347-1354, 2015.

[41] C. D. Katsis, N. Katertsidis, G. Ganiatsas, and D. I. Fotiadis, "Toward emotion recognition in car-racing drivers: A biosignal processing approach," IEEE Transactions on Systems, Man, and Cybernetics: Systems, vol. 38, no. 3, pp. 502-512, 2008.

[42] A. Riener, A. Ferscha, and M. Aly, "Heart on the road: HRV analysis for monitoring a driver's affective state," in Proceedings of the 1st International Conference on Automotive User Interfaces and Interactive Vehicular Applications, pp. 99-106, September 2009.

[43] C. Oh, J. Choi, and S. Park, "In-depth understanding of lane changing interactions for in-vehicle driving assistance systems," International Journal of Automotive Technology, vol. 18, no. 2, pp. 357-363, 2017. 


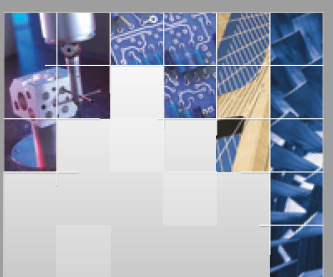

\section{Enfincering}
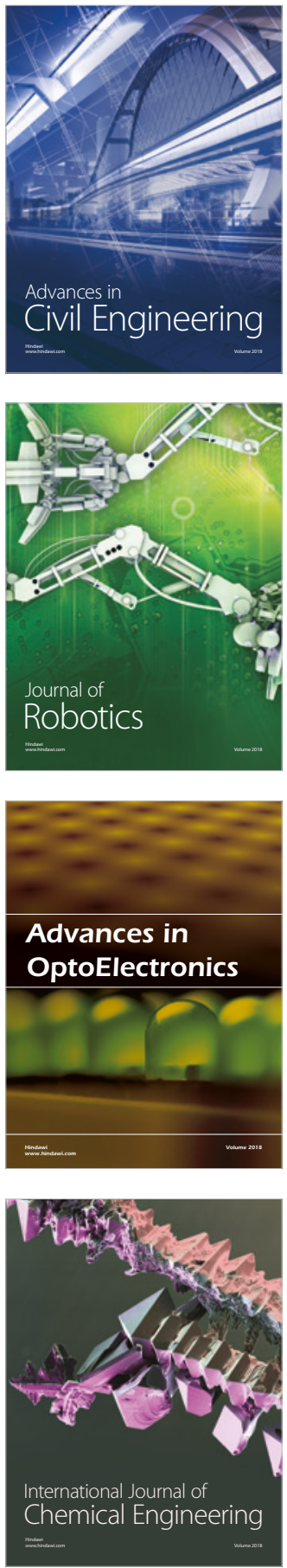

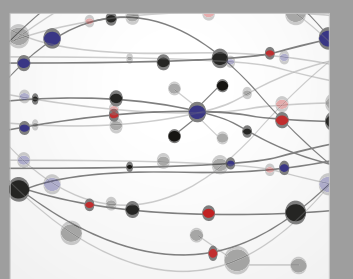

\section{Rotating \\ Machinery}

The Scientific World Journal

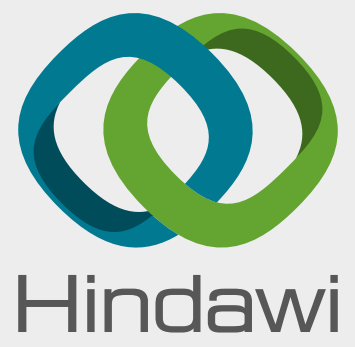

Submit your manuscripts at

www.hindawi.com
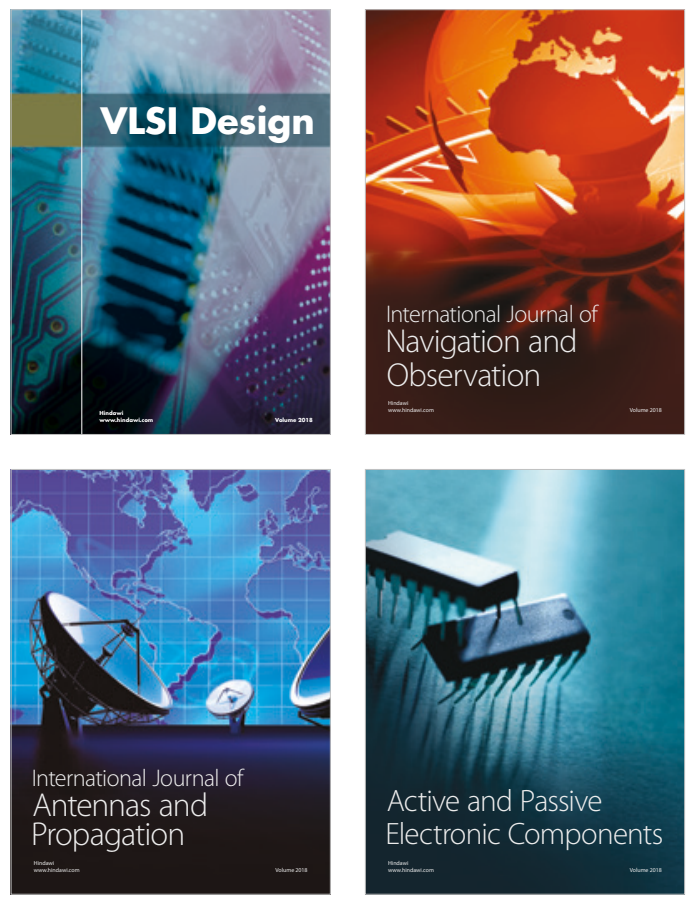
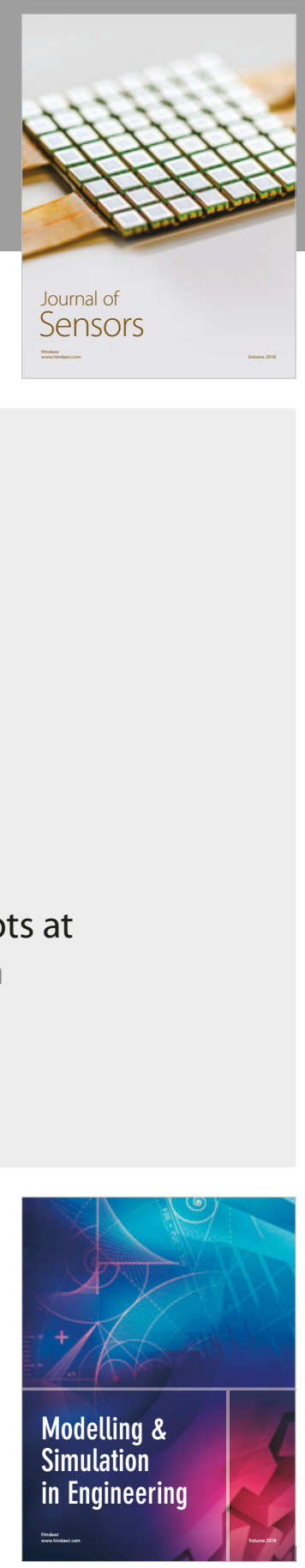

\section{Advances \\ Multimedia}
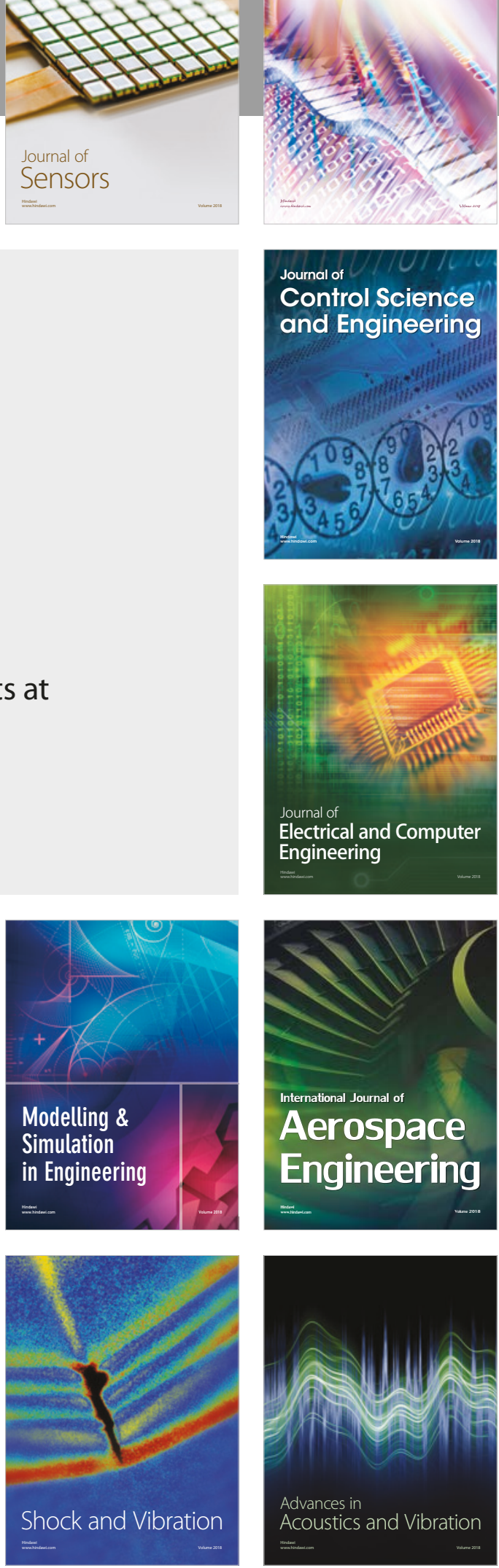